\title{
IS-2: Exploring modeling by programming: insights from numerical experimentation
}

\author{
Sean M. Laverty*, Brittany E. Bannish \\ Department of Mathematics \& Statistics, University of Central Oklahoma, Edmond, OK 73034 \\ slaverty@uco.edu
}

In this talk we provide an overview of the background necessary to use computational methods and numerical experimentation to pursue mathematical research. We highlight how numerical experimentation was critical to three undergraduate research projects: parasite transmission in feral cats, the role of protein regulation in establishing circadian rhythms, and treatment costs in an influenza outbreak. Use of computer code (written in R) is described for basic analysis of the models and we illustrate how nearly all of the figures presented in the chapter can be reproduced by the reader to encourage the learning of programming as they go along. We also propose ideas for extending the research projects presented in the text. Both deterministic and stochastic models are presented and the critical role of computational methods in obtaining solutions is illustrated. 